Original article

\title{
Quantitative determination of pupil by dynamic pupillometry using infrared videography - Role in evaluation of autonomic activity
}

\author{
A.V. Siva kumar ${ }^{\mathrm{a}}$, K.N. Maruthy ${ }^{\mathrm{b}}$, R. Padmavathi ${ }^{\mathrm{c}, *}$, B. Sowjanya ${ }^{\mathrm{d}}$, K. MaheshKumar ${ }^{\mathrm{e}}$ \\ ${ }^{a}$ Dept. of Physiology, Sri Ramachandra Medical College \& Research Institute, Porur, Chennai, Tamilnadu, 600116, India \\ ${ }^{\mathrm{b}}$ Dept. of Physiology, Narayana Medical College, Nellore, Andhra Pradesh, 524003, India \\ ${ }^{c}$ Dept. of Physiology, Sri Ramachandra Medical College, Porur, Chennai, Tamilnadu, 600116, India \\ ${ }^{\mathrm{d}}$ Dept. of Biochemistry, Narayana Medical College, Nellore, Andhra Pradesh, 524003, India \\ ${ }^{\mathrm{e}}$ Dept. of Physiology \& Biochemistry, Govt. Yoga and Naturopathy College \& Hospital, Chennai, Tamilnadu, 600104, India
}

\section{A R T I C L E I N F O}

\section{Keywords:}

Pupil diameter

Infrared videography

Validation

Pupillary light reflex

\begin{abstract}
A B S T R A C T
Introduction: Pupillometry is a simple, non-invasive method capable of measuring static and dynamic pupil response in several physiological and clinical conditions. The latest digital camera can be slightly modified to obtain real time pupil response under infrared videography and analyzed by image analysis. So the current study has undertaken to confirm characterization of the system by comparing with manual measurements in healthy volunteers.

Methods: This study was conducted in 40 healthy volunteers aged between 18 and 25 years. The system was calibrated by measuring the known length of 3 different lines for reliability. Later real time static pupil pictures were measured and compared with the manual examination of pupil determined by a ruled scale with $0.5 \mathrm{~mm}$ accuracy.

Results: The Image J software was precalibrated with known length and estimated $5 \mathrm{~mm}, 10 \mathrm{~mm}$ and $15 \mathrm{~mm}$ lines respectively. The results are $5.01 \pm 0.14 \mathrm{~mm}, 10.01 \pm 0.36 \mathrm{~mm}$ and $15.02 \pm 0.23 \mathrm{~mm}$. There were no significant differences observed between the values analyzed by two examiners. Later the results were compared between manual examination and real time image analysis.

Conclusion: The present study suggested that the static and dynamic pupil measurements with real time pupillometer are accurate and probably as reliable as those obtained by manual examination of the pupil using the flashlight test. This system is cost-effective, portable in nature which can produce authentic results of the pupil.
\end{abstract}

\section{Introduction}

Pupillometry is a convenient, feasible technique that is routinely used in the clinical set up as well as in research, to measure pupil size in various physiological and pathological conditions. ${ }^{1}$ The pupillary response can be influenced by exposure of the eye to bright light by means of pupillary light reflex, fixing gauge at a near point by accommodation reflex. ${ }^{2}$ These two reflexes lead to pupil constriction mediated through parasympathetic nerve activation (Occulomotor nerve). The Pupil is dilated in darkness, through sympathetic modulation. $^{3-5}$ So the pupil size for the light stimulus can be a sensitive indicator for evaluation of Autonomic nervous system activity. ${ }^{6-9}$ Apart from diagnosis of autonomic derangement, these quantified values of the pupil can help in traumatic brain injuries, pre-operative ophthalmic surgical procedures, and cortical nuclei damages. ${ }^{10}$ Various studies have shown that there are different automated pupillometers designed and established for pupil analysis. ${ }^{11}$ The infrared digital photographic devices and automated pupillometers are precise, accurate and repeatable for computation of static and dynamic measurements of the pupil diameter. ${ }^{12}$ However, these instruments are highly expensive, unaffordable, especially in poor and developing countries. Even though it is not feasible to use these devices for other setups such as semi-urban and rural OPDs and clinics. Nowadays the digitalization was profoundly developed and high-resolution web cameras become cost-effective and its usage also too easy. ${ }^{13}$ With the advancement of optics such as image stabilization, offline corrections and capturing good quality images has become comfortable and user-friendly. These cameras can also be employed to capture the pupil size and responses to the light stimulus that satisfy the necessities of clinical as well as research purposes. These are accessible and convenient tools that are commercially available in the

\footnotetext{
* Corresponding author.

E-mail addresses: reddy.sivakumar5@gmail.com (A.V. Siva kumar), dr.maruthy@gmail.com (K.N. Maruthy), rpadmavathi@sriramachandra.edu.in (R. Padmavathi), phanisowji@hotmail.com (B. Sowjanya),doctormahesh1985@gmail.com (K. MaheshKumar).
} 
market. However, they need to be redesigned to some extent as per our requirement to fulfil the above-mentioned purpose. So to obtain information about the pupil in resting state is feasible, effective and userfriendly technique. It is very essential to validate the modified system for accuracy, precision and reliability is essential for the critical judgment of the results. ${ }^{14}$ So the current study was aimed to validate the Personal Computer (PC) based real time pupillometer to record pupillary size under infrared digital videography which compared with the manual qualitative examination.

\section{Materials and methods}

\subsection{PC based real time pupillometer}

The pupillometer is a modified camera that objectively records static and dynamic pupil response. It is a simple, non-invasive tool to capture pupil in various light conditions. These measurements can be used to screen and diagnose different clinical diseases. The pupil does not respond to the infrared light which is beyond the visible spectrum (300-700 nm), because the photoreceptors respond to the visible spectrum of light which initiates pupillary light reflex. This helps to capture maximum dilated pupil which we cannot see by direct observation in darkness. But the pupil images are unable to record under infrared illumination with a normal camera. So the camera was customized and altered slightly to capture and appreciate maximum pupil with infrared light (fig. 1).

\subsection{Design of pupillometer}

The web camera $($ Technotech ZB V90 WEBCAM- $640 \times 380$ ) slightly altered to record under infra-red illumination where the infra-red filter was removed from optics. The camera was fixed to a virtual reality box (VR box) to avoid the influence of external ambient fluctuations on the recordings. The spatial distance of $7 \mathrm{~cm}$ was maintained from the anterior curvature of the eye to avoid accommodation reflex. It was also connected with a microcontroller-based electronic circuitry to control the standard intensity of Infra-red LED with $850 \mathrm{~nm}(2 \times 5 \mathrm{~mW}$ continuous) and white light stimulus ( $5 \mathrm{~mm} \mathrm{LED} \mathrm{-} 5 \mathrm{~mW} / 2000 \mathrm{~ms}$ ) Fig. 2. The authors of current paper had already established the methodological protocol to analyze pupil diameter recorded by the same instrument. ${ }^{15}$

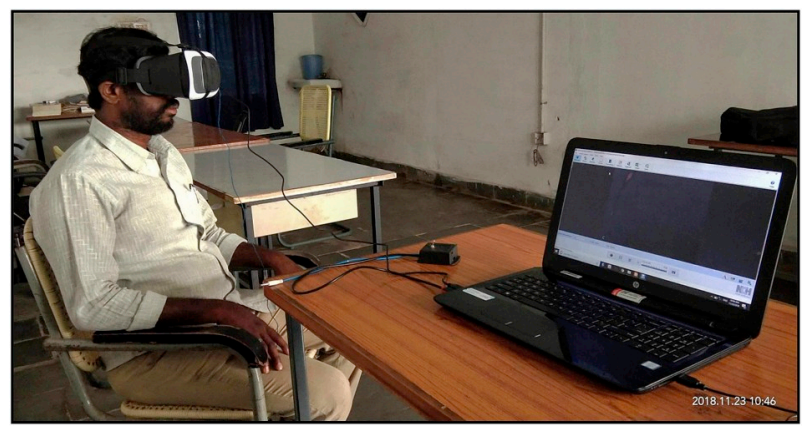

Fig. 2. The position of VR box, Illumination controlling electronic circuit.

\subsection{Calibration}

Initial characterization of the pupillometer was evaluated with preliminary calibration steps. The known distances of 3 straight lines with $5 \mathrm{~mm}, 10 \mathrm{~mm}$ and $15 \mathrm{~mm}$ were drawn on a curved surface of a thick paper sheet with a ruled scale respectively. This sheet was aligned in the exact position in the system where the anterior surface of the eye can be seen from the device. The picture of the same sheet was captured repeatedly by the web camera through Debut software (Version 5.09, NCH software) which is an open-source application for noncommercial purpose. The captured image was subjected to an image analysis software i.e. Image $J$ (Image $J$ 1.52a, National Institutes of Health, USA) to measure the lines instantly. Calibration steps were followed with the true known size of the 3 lines in the image (see Fig. 2).

\subsubsection{Intrameasurer variability}

To check Intra measurer variability for recurrent measurements, each line was measured repeatedly in the captured images. The same procedure was performed for different lengths include $5 \mathrm{~m}, 10 \mathrm{~mm}$, and $15 \mathrm{~mm}$. These results were compared with the manual length of lines drawn by the ruled scale. They were analyzed by estimating coefficient variation $(\mathrm{CV} \%)$.

\subsubsection{Intermeasurer variability}

To check inter measurer variability, the above-mentioned procedure was repeated by another expert who is familiar with these techniques. The data obtained from two examiners were statistically compared using the parametric test, i.e. Paired't' test for inter measurer variability (Table. 2).

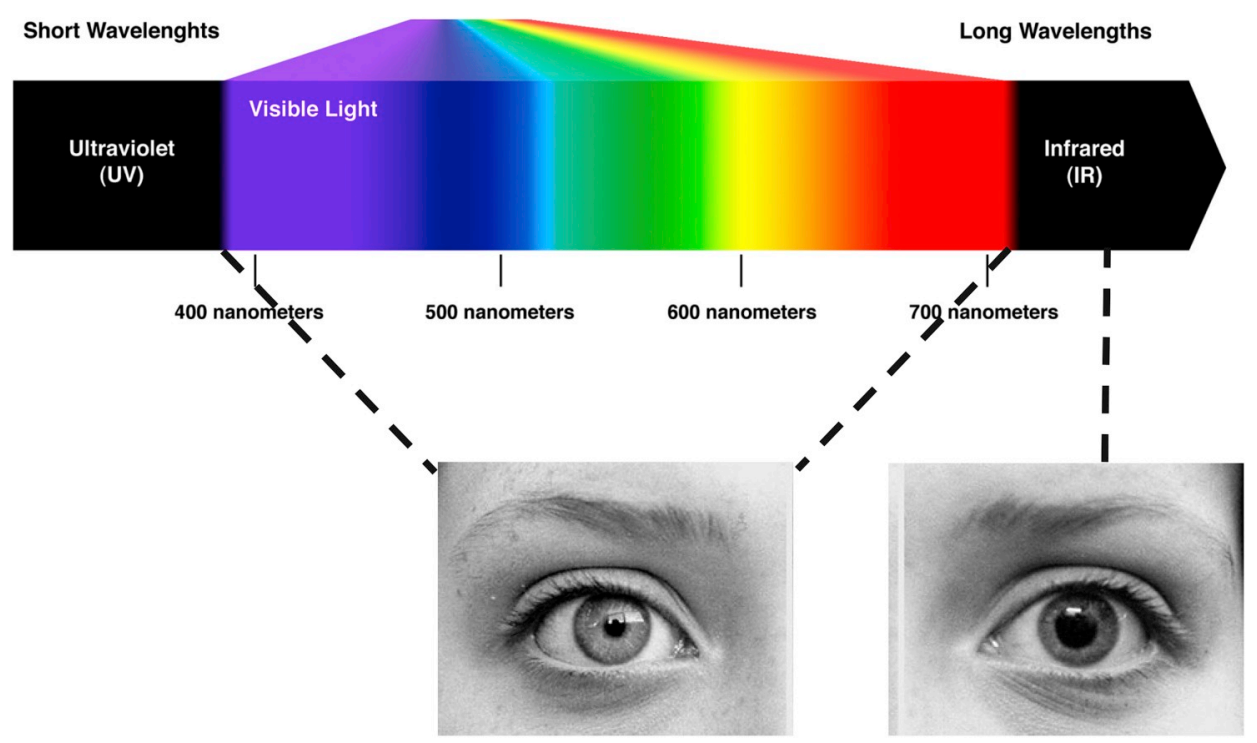

Fig. 1. Effect of visible and infrared light on the size of the pupil. 
Table 1

Intrameasurer reliability of the real time measured repeatedly by two examiners.

\begin{tabular}{|c|c|c|c|c|c|c|}
\hline \multirow[t]{2}{*}{ Known length } & \multicolumn{3}{|l|}{ 1st examiner } & \multicolumn{3}{|l|}{ 2nd examiner } \\
\hline & $5 \mathrm{~mm}$ & $10 \mathrm{~mm}$ & $15 \mathrm{~mm}$ & $5 \mathrm{~mm}$ & $10 \mathrm{~mm}$ & $15 \mathrm{~mm}$ \\
\hline Mean \pm SD & $5.01 \pm 0.14$ & $10.01 \pm 0.36$ & $15.02 \pm 0.23$ & $5.07 \pm .14$ & $9.99 \pm .26$ & $15.01 \pm 0.21$ \\
\hline Mean of differences & 0.014 & 0.014 & 0.011 & 0.07 & 0.005 & 0.011 \\
\hline Median & 5.07 & 10.17 & 15.06 & 5.10 & 9.88 & 15.03 \\
\hline$\% \mathrm{CV}$ & 2.99 & 3.66 & 1.59 & 3.51 & 2.66 & 1.42 \\
\hline $95 \%$ CI & $4.90-5.12$ & $9.75-10.28$ & $14.85-15.19$ & $4.95-5.20$ & $9.80-10.19$ & $14.86-15.16$ \\
\hline
\end{tabular}

Table 2

Intermeasurer variability of the measurements between two examiners.

\begin{tabular}{lllll}
\hline Calibration & & $5 \mathrm{~mm}$ & $10 \mathrm{~mm}$ & $15 \mathrm{~mm}$ \\
\hline \multirow{2}{*}{ 1st examiner } & Mean \pm SD & $5.01 \pm 0.14$ & $10.01 \pm 0.36$ & $15.02 \pm 0.23$ \\
& Std. Error & 0.04 & 0.11 & 0.07 \\
2nd examiner & Mean \pm SD & $5.07 \pm 0.14$ & $9.99 \pm 0.26$ & $15.01 \pm 0.21$ \\
& Std. Error & 0.05 & 0.08 & 0.06 \\
p-value & & 0.50 & 0.91 & 0.96 \\
\hline
\end{tabular}

Table - 3

shows a comparison of circular object measurement between manual (Ruler) and pupillometer and between two examiners.

\begin{tabular}{llll}
\hline & 1st examiner & 2nd examiner & p-value \\
\hline Manual measurement & $9.0 \mathrm{~mm}$ & $9.0 \mathrm{~mm}$ & 0.99 \\
Image analysis & $9.465 \mathrm{~mm}$ & $9.460 \mathrm{~mm}$ & 0.90 \\
p-value & 0.90 & 0.90 & \\
\hline
\end{tabular}

Later the calibration was extended to a circular object (sticker) where it was placed over closed eyelid. Because the pupil shape is oval to round located in the anterior aspect of the eye. The circular object stick on the closed eyelid resembles the exact pupil location. This step was followed to establish the morphometric variation of distance between pupil and camera. A still picture of the sticker was taken and repeated the above procedure with two examiners after manual measurement of the sticker diameter (Table. 3 ).

The initial calibration steps were suggested that the pupillometer produces reliable, accurate values through image analysis. However the lines were measured by selecting the distance appeared in the images manually with the cursor (Computer mouse). But the pupil size was accurately measured through a java based plugin code was developed which can measure either single or batch of images. The error can be minimized using automatic batch processing.

\subsection{Permission}

After the preliminary evaluation of the pupillometer, the study was extrapolated to the healthy volunteers to measure real time pupil recording and measurement. The entire protocol was reviewed and approved by the Institutional ethical committee (REF: IEC - NI/18/JAN/ 63/10), Sri Ramachandra Medical College and Research Institute, Chennai, and the Institutional ethics committee, Narayana Medical College, Nellore in accordance with the Declaration of Helsinki.

\subsection{Study population}

40 healthy volunteers aged between 18 and 25 years with no history of ocular pathology or ocular trauma were included in the study. Among these participants 25 were females and 15 were males. They were also inquired about the clinical history of any diseases, lifestyle behaviour. The subjects are medical students and other technical staff of the hospital premises who are acquainted with the investigator. The subjects with the history of known acute or chronic medical illness, chronic smokers, alcoholics and intake of centrally acting drug patients were excluded from the study. The subjects were recruited through non - random sampling technique (Convenient sampling). Written informed consent was obtained from all the participants after explaining the complete nature of the experiment. The refractive errors of the subjects were not noticed. However refractive errors do not influence pupil size. The included subjects were followed a few specific guidelines before the data acquisition because the pupil is supplied by ANS subdivisions. So it can be influenced by various factors that alter the resting pupil dimensions. The participants were directed to take normal food, to maintain good sleep pattern and to abstain centrally acting beverages like caffeine that may influence the measures carried out. The subjects were also instructed not to execute any physical exercise $24 \mathrm{~h}$ before the evaluations. All the static and dynamic recordings were performed in morning hours in the Research lab, Department of physiology in Narayana Medical College, Nellore. The participants were informed to stop physical movements during the data acquisition to reduce their activity and stay calm and relax. Participants were also instructed to avoid blinking and eyeball movements before, during and after visual stimulation, moments in any angle might alter the pupil size in a video graphic analysis. The saccadic moments could also confound the test results. The final validation of the pupillometer was determined by executing real time recording of the pupil in a static and dynamic state.

\subsection{Static pupil evaluation}

The real time monocular static pupil pictures were taken separately in both eyes with the help of web camera, followed by physical measurement of the same pupil in each participant in dim illumination in two sessions (Torch light \& Indefinite darkness) using a ruled scale with an accuracy of $0.5 \mathrm{~mm}$. The examiner was stood on one side of the subject and kept the scale away from the visual axis to measure the pupil. The manual static pupils were measured at a one-inch distance from the anterior surface of the eye, and the subject is directed to see a distant object to avoid accommodation reflex. It was difficult for a few subjects to measure pupil size as the iris was dark. All the recordings were executed with a constant dim illumination to avoid the fluctuations which might affect pupil size. Later the static pictures were taken with the pupillometer, were measured from the captured images with help of Image $\mathrm{J}$ software as mentioned in the calibration procedure (Figs. 3 and 4). The pupil diameter was measured using java based macro code that determines absolute outline of pupil. The data obtained by manual and image analysis techniques were compared to evaluate the accuracy of the static measurements of the designed pupillometer.

\subsection{Dynamic pupil evaluation}

The dynamic pupil response was examined with the swinging flashlight test to observe pupillary light reflex. The examination of the pupil to a light stimulus was performed in the non-accommodative eye by instructing the subject to fix a distant target. The examiner (clinician) directed light into eye, one inch away from the eye for $2 \mathrm{~s}$. The response was observed in both eyes of each participant for direct and consensual reflex. 


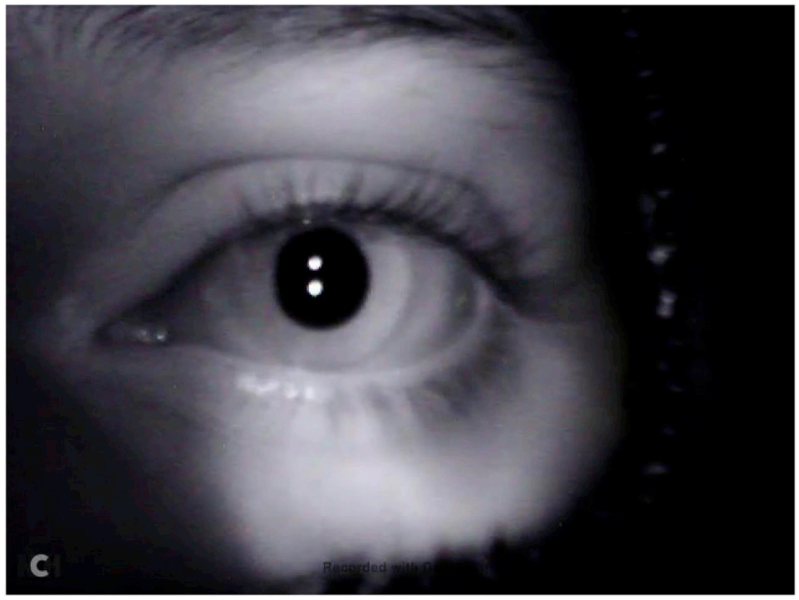

Fig. 3. Real time Pupil dilation (Mydriasis) under IR illumination.

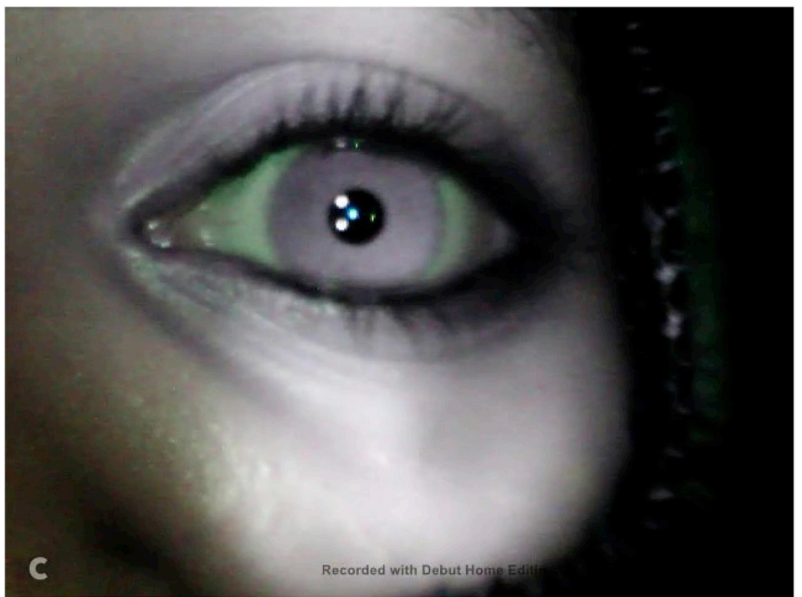

Fig. 4. Real time Pupil constriction (Miosis) under white light illumination.

The participants were immediately subjected to subsequent videographic recording of pupillary light reflex using dynamic pupillometry. After $5 \mathrm{~min}$ of dark adaptation, monocular Pupillometry was recorded for $10 \mathrm{~s}$ with controlled illumination within the box that was positioned to the head of the subject to avoid the influence of external illumination. The videography was performed using debut software as mentioned in the calibration protocol. Dynamic pupillometry was recorded in total darkness with the illumination of IR LED $(2 \times 10 \mathrm{~mW})$ to record pupil dilation followed by a flash of white light ( $5 \mathrm{~mm} \mathrm{LED} / 2000 \mathrm{msec}$.) to record pupillary constriction. Stimulus application and data acquisition were controlled by electronic circuit fixed a little far away from the web camera. This was video graphed through modified web camera with the frame rate of 30 frames/sec with a resolution of $33.3 \mathrm{msec} /$ frame. The video was cleaved into 300 pictures with the help of Video to JPG Converter (Ver: 5.0.101.20). These frames were subjected to an image analysis software (ImageJ ver.1.43u National Institute of Health, USA). A macro code was designed for analysis of all images which run as a batch file to measure baseline pupil diameter (BPD), Minimum pupil diameter (MPD). The velocities like maximum constriction velocity (MCV), maximum re-dilation velocity (BRDV) were calculated with the help of a number of frames and change in diameter for each frame (Table 4).

\section{Statistical analysis}

Statistical analysis was carried out using SPSS 17 and data was represented as mean and SD. Normality of data was tested using the Kolmogorov-Smirnov test. A $p$-value of $>0.05$ indicated normal
Table 4

Dynamic pupil evaluation (Quantitative Pupillary light reflex).

\begin{tabular}{llll}
\hline Dynamic pupil parameters & Right eye & Left eye & p-value \\
\hline BPD & $4.56 \pm 0.45 \mathrm{~mm}$ & $4.39 \pm 0.50 \mathrm{~mm}$ & 0.30 \\
MCV & $2.89 \pm 0.39 \mathrm{~mm} / \mathrm{s}$ & $2.97 \pm 0.42 \mathrm{~mm} / \mathrm{s}$ & 0.54 \\
MPD & $2.10 \pm 0.38 \mathrm{~mm}$ & $2.12 \pm 0.35 \mathrm{~mm}$ & 0.86 \\
MRDV & $1.93 \pm 0.31 \mathrm{~mm} / \mathrm{s}$ & $1.89 \pm 0.33 \mathrm{~mm} / \mathrm{s}$ & 0.65 \\
\hline
\end{tabular}

BPD- Baseline pupil diameter, MCV- Maximum constriction velocity, MPDMinimum pupil diameter, MRV- Maximum redilation velocity.

Gaussian distribution. The Paired't' test was performed for intraobserver variability.

\section{Results}

This is a cross sectional study has taken over in 40 healthy subjects in their both eyes. The mean age of the subject $19.68 \pm 0.72$ yrs. Among these participants 25 are females and 15males. It was difficult to estimate some of the pupil images in sequence of frames due to eye blinking and deviation from the visual axis. 20 subjects were participated for static pupil evaluation of the both eyes in two different illumination measured by two methods. Remaining were subjected to record pupillary light reflex using infrared videography. The static pupil was measured for right pupil is $4.09 \pm 0.50 \mathrm{~mm}$, left pupil is $3.84 \pm 0.84 \mathrm{~mm}$ under IR illumination and right pupil $2.23 \pm 0.48 \mathrm{~mm}$, left pupil is $2.21 \pm 0.63 \mathrm{~mm}$ under bright light illumination (Flash of white light). The dynamic pupillometry was used to record pupillary light reflex which can be divided into BPD, MCV, MPD and MRV. The MCV and MRV are the velocities were calculated based on change in diameter with frame rate. We have faced difficulty when we were measureds pupil in darkness.

\section{Discussion}

The current study was aimed to evaluate the precision and accuracy of the PC based real time pupillometer. The stepwise validation of strategic techniques followed in this protocol that revealed the characterization of the entire system. The results in each phase of validation displayed the reliability and consistency of the system.

The initial calibration was started to establish intraobserver variability for the analysis of the repeated measurements to confirm consistent reproducibility of the system (Table. 1). There were no significant differences between the two values and for any recurrent measurements of 3 lines from the true length of the lines. However the CV\% is more than 1 which indicates large variability. But it is a relative measure of variability which is not reliable. This preliminary data suggests that the values produced by the pupillometer are trustworthy and reliable. The data between two experts were not statistically significant for the measurements of the 3 lines which reveals that the interobserver variability is also negligible (Table. 2). However, the position of the thick paper sheet that contains the 3 different lines is constant. But the anatomical location of the pupil distance from the camera may not be equal for every individual. So the error might create with pupillary distance variability was established through the circular object determination with the pupillometer. In addition, the circular object was selected due to the pupil is identical in shape which might produce similar results (Table. 3 ). There was no significant variation for the values of the circular object between manual scaling and pupillometer, which was kept on the closed eyelid. So the preliminary steps of calibration gave us favourable and promising results for the pupillary evaluation. Various studies have shown the different modalities of validation protocols for static and dynamic pupil responses ${ }^{16-19}$ This is the first paper describes the initial calibration of the device and offline character of the software for the reliable results to the best of our knowledge.

The real time recording of a pupil in static and dynamic state values were compared with manual examinations. The tonic contractions of the pupil fluctuate continuously (19). They can be influenced by various 
factors like illumination, cognition, attention span, stress and different kinds of emotions. ${ }^{20-22}$ The data of these measurements were not significantly different between the two methods. This suggests that the images taken by the web camera and analyzed by Image $J$ are measuring accurately and precisely, the variations in repeatable measurements are not significant. Nefati Kiylığlu et al. had also worked on the customized pupillometer to evaluate pupil size using matlab and evaluated pupil paradigms at different time periods. ${ }^{23}$ The dynamic pupillometry quantitative values of pupillary light reflex were descriptively mentioned as velocities which reflect the fine autonomic balance. However there is no normative data for these dynamic responses of pupillary light reflex. So we compared inter pupil difference for static and dynamic parameters which was statistically insignificant. The robustness of this methodology was explained by abovementioned calibration and real time results which can be used for wide variety of clinical applications.

\section{Advantages of real time pupillometer}

The complete dilated pupil cannot be appreciated and measured by manual method in total drakness. However the dilated pupil can be visualize, record and measured using real time pupillometer under infrared illumination. The IR LEDs which were used in this setup are not injurious to the eye and other appendages. The images of real time pupillometer helps to identify precise pupil and iris borders even though they are dark. The Image $\mathrm{J}$ software is precalibrated with the known length and given less variability for repeatable measurements. The images can be captured in different illuminations (white and IR) which can measure both extreme diameters of pupil. The illumination can be controlled accurately using electronic circuit to maintain constant strength of stimulus. The manual examination of pupil was tedious, may led to accommodation reflex if the scale move towards the pupil. The visual axis may also influence the size of pupil as it was arranged in curvature. So the results explored from these images using Image $\mathrm{J}$ are accurate and reliable for pupil diameter. ${ }^{24}$

\section{Limitations}

There are some limitations for pc based real time pupillometry for pupil measurements. The data of this device was not compared with any other gold standard device due to its unavailability. However we had tried our best to prove the characterization of the device with our limited resources. The monocular pupillary recording only can execute in a single session. There are multiple softwares to be used to achieve the results which can be evaluated only in offline. It is also time consuming, tedious which requires basic fundamentals on computer applications. We are in the process of developing a real-time binocular pupillometer for analysis of pupil character.

\section{Conclusion}

The results of this study recommend that the estimation of dynamic pupillometry by simple infrared digital videography with the web camera for pupil analysis is the reliable one and compare with the clinical examination which has more advantages such as very low cost, portable in nature and simple to use either laboratory or in the fieldoriented study. The pupil dimensions help to screen and diagnose in multiple fields of medicine, and our customized pupillometer may be useful as a noninvasive diagnostic tool.

\section{Funding}

The authors declared that there is no financial support received for this study.

\section{Declaration of competing interest}

The authors declare that there is no conflict of interests regarding the publication of this paper.

\section{Acknowledgement}

We would like to express our sincere thanks to the Sri Ramachandra Medical College and Research Institute, Chennai and Narayana Medical College, Nellore for approving the study design and for assisting the project during data collection and constant support throughout the study period.

\section{References}

1. D Du R, Meeker M, Bacchetti P, Larson MD, Holland MC, Manley GT. Evaluation of the portable infrared pupillometer. Neurosurgery. 2005 Jul 1;57(1):198-203.

2. Mathôt S. Pupillometry: psychology, physiology, and function. J. Cognit. 2018 Feb $21 ; 1(1)$.

3. Mathôt S, Van der Stigchel S. New light on the mind's eye: the pupillary light response as active vision. Curr Dir Psychol Sci. 2015 Oct;24(5):374-378.

4. Ajasse S, Benosman RB, Lorenceau J. Effects of pupillary responses to luminance and attention on visual spatial discrimination. $J$ Vis. 2018 Oct 1;18(11):6.

5. van der Wel P, van Steenbergen H. Pupil dilation as an index of effort in cognitive control tasks: a review. Psychon Bull Rev. 2018 Dec 1;25(6):2005-2015.

6. Pittasch D, Lobmann R, Behrens-Baumann W, Lehnert H. Pupil signs of sympathetic autonomic neuropathy in patients with type 1 diabetes. Diabetes Care. 2002 Sep 1;25(9):1545-1550.

7. Smith SE, Smith SA, Brown PM, Fox C, Sönksen PH. Pupillary signs in diabetic autonomic neuropathy. Br Med J. 1978 Sep 30;2(6142):924-927.

8. Wang Y, Zekveld AA, Naylor G, et al. Parasympathetic nervous system dysfunction, as identified by pupil light reflex, and its possible connection to hearing impairment. PloS One. 2016 Apr 18;11(4):e0153566.

9. Muppidi S, Adams-Huet B, Tajzoy E, et al. Dynamic pupillometry as an autonomic testing tool. Clin Auton Res. 2013 Dec 1;23(6):297-303.

10. Hall C, Chilcott R. Eyeing up the future of the pupillary light reflex in neurodiagnostics. Diagnostics. 2018 Mar;8(1):19.

11. Chaglasian EL, Akbar S, Probst LE. Pupil measurement using the Colvard pupillometer and a standard pupil card with a cobalt blue filter penlight. $J$ Cataract Refract Surg. 2006 Feb 1;32(2):255-260.

12. Boev AN, Fountas KN, Karampelas I, et al. Quantitative pupillometry: normative data in healthy pediatric volunteers. J Neurosurg Pediatr. 2005 Dec 1;103(6):496-500.

13. Mariakakis A, Baudin J, Whitmire E, et al. PupilScreen: using smartphones to assess traumatic brain injury. Proceed. ACM Interact. Mobile Wear. Ubiquitous Technol. 2017 Sep 11;1(3):81.

14. Mease L, Sikka R, Rhees R. Pupillometer use: validation for use in military and occupational medical surveillance and response to organophosphate and chemical warfare agent exposure. Mil Med. 2018 Mar 14;183(9-10):e291-e296.

15. Kumar AS, Padmavathi R, Maruthy KN, Sowjanya B, Kumar K. An innovative technique to evaluate quantitative pupillary light reflex by dynamic pupillometry using infrared videography. J Clin Diagn Res. 2019 Apr 1;13(4).

16. Zhao W, Stutzman S, DaiWai O, Saju C, Wilson M, Aiyagari V. Inter-device reliability of the NPi-100 pupillometer. J Clin Neurosci. 2016 Nov 1;33:79-82.

17. Zavagno D, Tommasi L, Laeng B. The eye pupil's response to static and dynamic illusions of luminosity and darkness. i-Perception. 2017 Aug;8(4) 2041669517717754

18. Taylor WR, Chen JW, Meltzer H, et al. Quantitative pupillometry, a new technology: normative data and preliminary observations in patients with acute head injury. $J$ Neurosurg. 2003 Jan 1;98(1):205-213.

19. Iacoviello D. Analysis of pupil fluctuations after a light stimulus by image processing and neural network. Comput Math Appl. 2007 Apr 1;53(8):1260-1270.

20. Zafar SF, Suarez JI. Automated pupillometer for monitoring the critically ill patient: a critical appraisal. J Crit Care. 2014 Aug 1;29(4):599-603.

21. Litvan I, Saposnik G, Maurino J, et al. Pupillary diameter assessment: need for a graded scale. Neurology. 2000 Jan 25;54(2):530.

22. Twa MD, Bailey MD, Hayes J, Bullimore M. Estimation of pupil size by digital photography. J Cataract Refract Surg. 2004 Feb 1;30(2):381-389.

23. Kıylığlu N, Kılıç MA, Kocatürk T, Özkan SB, Bilgen M. A custom-made pupillometer system for characterizing pupillary light response. Turkish J Orthod. 2018 Aug;48(4):185.

24. Ramadevi PP, Maruthy KN, Padmavathi R, Kumar AV, Kareem SK. Evaluation of static pupil size using digital web camera through image analysis. Int. J. Physiol. 2019;7(3):7-11. 